\title{
LIDERAZGO EN ENFERMERIAA DESDE LA PERSPECTIVA DE SUS SEGUIDORES
}

\section{CARE OF THE PERSON WITH MOTOR DISABILITIES}

Recibido enero 2016 Aceptado abril 2016

Correspondencia: Albatros 300 Interior 3-C Colonias las Gaviotas. C.P.78340 Tel: 4441000215 cel. 4441264874 E-mail: tlaurasoledad@yahoo.com

\section{Autores:}

Torres- Flores Soledad

Universidad Autónoma de San Luis Potosí

Unidad de Posgrado e Investigación

Rodríguez Padilla Ma. Elsa

Universidad Autónoma de San Luis Potosí

Unidad de Posgrado e Investigación

Andrade Cepeda Guadalupe

Universidad Autónoma de San Luis Potosí

Unidad de Posgrado e Investigación

Palabras clave: perfil de liderazgo en enfermería; liderazgo de Rango Total en enfermería

Key words: Profile of leadership in nursing; full-range leadership in nursing 


\section{RESUMEN}

Objetivo: Analizar el perfil de liderazgo de los directivos en enfermería desde la perspectiva de sus seguidores.

Material y Métodos: Estudio cuantitativo, descriptivo y transversal, se realizó a 27 directivos de enfermería, se aplicó el cuestionario Multifactor Leadership Questionary (MLQ) del liderazgo de Rango Total, que mide 3 perfiles de liderazgo: Transformacional, Transaccional y Laissiez- Faire y las variables de resultado: esfuerzo extra, efectividad y satisfacción.

Resultados: En el perfil de liderazgo en Laissiez faire, calificó $67 \%$ la jefa de enfermeras, $43 \%$, las supervisoras, en liderazgo transaccional, correspondió 42\% para los jefes de servicio y la combinación de perfiles de liderazgo transaccional y Laissiez faire, la obtuvieron $54.4 \%$ en los tres niveles directivos, de las variable de resultado: solo en efectividad se ubicaron la jefe y jefes de servicio. Conclusiones: por perfiles de liderazgo que predominaron de Laissiez faire y transformacional, se puede considerar que son líderes visionarios que también actúan de manera situacional en algunas circunstancias a fin obtener de sus seguidores los mejores resultados, por lo tanto medir el liderazgo de rango total da un panorama general de acurdo a cada situación.

Palabras clave: perfil de liderazgo en enfermería; liderazgo de Rango Total en enfermería.

\section{ABSTRACT}

Objective: To analyze the profile of leadership of managers in nursing from the perspective of their followers.

Material and Methods: quantitative study, descriptive and cross-sectional, was performed in 27 directors of nursing, the questionnaire was applied (MLQ Multifactor Leadership Questionary) of the leadership of Total Range, which measures 3 transformational leadership: profiles, transactional and Laissiez- faire and the outcome variables: extra effort, effectiveness and satisfaction.

Results: In the profile of leadership in Laissiez faire, described $67 \%$ the head nurse, 43\%, supervisors, in transactional leadership, was $42 \%$ for the heads of service and the combination of profiles of transactional leadership and Laissiez faire, obtained $54.4 \%$ in the three levels of management, of the outcome variable: only in effectiveness was placed the head and heads of service. Conclusions: by profile leadership that predominated of Laissiez faire and transformational, can be considered that are visionary leaders who also act of situational manner in some circumstances to obtain their followers the best results, therefore measure the leadership of total range gives an overview according to each situation.

Keywords: Profile of leadership in nursing; full-range leadership in nursing. 


\section{INTRODUCCIÓN}

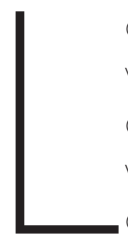

as organizaciones en la actualidad se desenvuelven en ambientes de gran turbulencia y dinamismo; por lo que es necesario adaptarlas y transformarlas de acuerdo con las exigencias y contingencias del medio ambiente cambiante?

Una de estas organizaciones son las instituciones de salud que adoptan una estructura formal diseñada para que en la división del trabajo y la especialización sea responsable de un aspecto particular en el funcionamiento del hospital, para lo cual las tareas se subdividen en departamentos. Por lo que respecta al departamento de enfermería, la organización de su servicio en las instituciones de salud, resulta ser un elemento indispensable para que se garantice una óptima y continua atención a los usuarios. ${ }^{2}$

Lo anterior debido a que el departamento de enfermería labora los 365 días del año las 24 horas del día, que para cubrir administrativamente un día laboral se distribuye en tres turnos; matutino, vespertino y nocturno, su estructura organizacional está diseñada jerárquicamente y bien definida para precisar los puestos, las obligaciones y la responsabilidad de cada integrante; en la cúspide de la misma se encuentra el jefe de enfermería que como directivo desempeña funciones de planeación, organización, dirección y control, el liderazgo por lo tanto forma parte de su labor y a su vez el equipo de gestión, le corresponden funciones como el dirigir y coordinar las unidades y servicios así como evaluar las actividades de enfermería. ${ }^{3}$

Es de importancia para enfermería analizar el liderazgo, porque como es bien sabido, en una institución de salud, el personal de enfermería representa más del 50\% del total de su plantilla, y al conformar la mayor parte del recurso humano, su papel en el sistema de salud es definitivo, pues liderea cambios esenciales al interior de la organización e influye en la definición y ejecución de objetivos organizacionales al ser un gran conocedor de las necesidades individuales y colectivas de salud. ${ }^{4}$

\section{OBJETIVO}

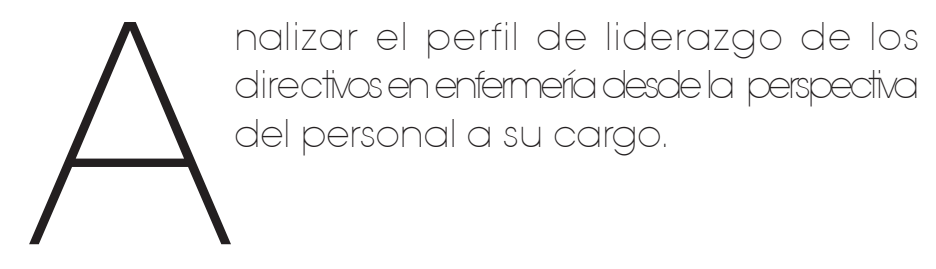

\section{MATERIALES Y MÉTODOS}

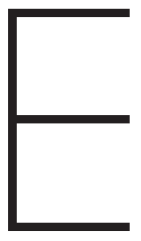

studio con enfoque cuantitativo, descriptivo y transversal, de 117 elementos de enfermería de un Hospital Privado en San Luis Potosí, la población de estudio se conformó con 27 directivos de enfermería (Jefa de Enfermeras, supervisores de enfermería, jefes de servicio) y los que se nominaron como seguidores fue el personal operativo de los diferentes servicios con 90. El liderazgo se midió de acuerdo a la teoría de Rango Total, propuesta por Bass y Avolio, en la que estructura el cuestionario "Multifactor Leadership Questionnaire (MLQ)" Versión visto por otros. ${ }^{5}$ estructurado en nueve dimensiones, para determinar 3 perfiles de liderazgo: transformacional, transaccional y Laissiez-faire, y tres variables de resultado: esfuerzo extra, efectividad y satisfacción, que representan la influencia del líder sobre sus seguidores, ${ }^{\circ}$ Contenidas en 75 items, medido con una escala ordinal tipo Likert, con 5 opciones de respuesta (O nunca; 1 rara vez, 2 a veces, 3 a menudo, 4 siempre).

Para el procesamiento y análisis de los datos se apoyó en paquete estadístico SPSS v 20, se utilizó estadística descriptiva: frecuencias absolutas y relativas. Para obtener los perfiles de liderazgo, se sumaron las 12 dimensiones y se determinaron los rangos de ponderación, bajo, medio y alto, de igual manera para las tres variables de resultado; esfuerzo extra, efectividad y satisfacción.

El estudio se realizó conforme a lo que dispone el Reglamento de la Ley General de Salud en Materia de Investigación.? Se contó con la aprobación de

\section{8}

LMN /Whw Enf Neurol Vol.15. No. 2 mayo - agosto 2016 
los Comités de Ética e Investigación de la Facultad de Enfermería de la Universidad Autónoma de San Luis Potosí, y del comité de ética de la institución en donde se realizó el estudio se solicitó autorización de los participantes a través de la firma del consentimiento informado y en todo momento se respetó la dignidad, privacidad, bienestar y los derechos de los participantes.

\section{RESULTADOS}

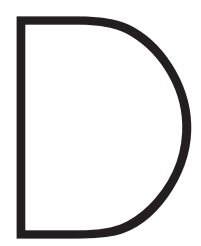

e la población de estudio 96.3\% fueron mujeres, $77.8 \%$ cuenta con nivel académico de Licenciatura en Enfermería, el turno con el mayor número de encuestados fue el vespertino con $27.8 \%$, el servicio de hospitalización ubicó el mayor número de participantes con 26.7\%.

En cuanto al perfil de liderazgo se encontró que la jefa de enfermeras se situó en el perfil transformacional en nivel medio, de igual manera para el perfil transaccional y alto para el perfil Laissiez-faire. (Cuadro 1). En las supervisoras y Jefes de Servicio se encontró perfil de liderazgo medio para el liderazgo transformacional y para perfil transaccional y alto para perfil de liderazgo Laissiez-faire (Cuadro 2). En las variables de resultado, en esfuerzo extra y satisfacción, la jefa de enfermeras obtuvo puntajes altos, Las supervisoras de enfermería alcanzaron en efectividad y satisfacción puntaje alto; en jefes de servicio lograron los más altos puntajes la satisfacción y esfuerzo extra(Cuadro 4).

\section{DISCUSIÓN}

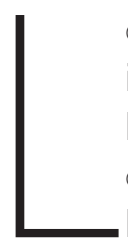

os resultados de esta investigación permitieron identificar el perfil de liderazgo visto desde la perspectiva del personal operativo, de acuerdo a la teoría del rango total de liderazgo, liderazgo mucho más amplia de tal modo que en este estudio se identificó que aunque predomino el perfil de liderazgo Laissiez faire en los tres niveles investigados: jefe, supervisores y jefes de servicio y que en las variables de resultado se encontró en la jefe de enfermeras un nivel alto de esfuerzo extra y satisfacción que sugieren que las acciones del líder provocan mayor participación de los seguidores en cuanto a empuje en su trabajo cotidiano, estimula a participar activamente en cuanto se necesite de su colaboración, esto sería un factor a favor del perfil de liderazgo Laissiez faire, de manera que cuando la jefe requiere el esfuerzo su equipo atiende. En los supervisores y jefes de piso se encontró de igual manera que predominaba la variable satisfacción; que según Bass y Avolio indica que las acciones del líder provocan gratificación en el grupo de trabajo, los seguidores se sienten muy bien con las decisiones del líder y se crea un clima organizacional sano para el buen desarrollo de las actividades. La otra dimensión de la teoría de rango total que resultó en nivel medio en los tres niveles fue l a del perfil transaccional, resultados que coinciden con los del Rozo, Abaunza; Berdecía 8, 9. de manera que se da evidencia que el departamento de enfermería cuenta con líderes que inspiran y facilitan el cambio ya que el líder transaccional es aquel que motiva a los seguidores para un mejor rendimiento en el servicio, se distingue porque motiva a trabajar por metas trascendentales y despierta altos niveles de necesidades de actualización, más bien que por un auto interés inmediato. Con estos hallazgos se puede decir que el departamento de enfermería cuenta con líderes que facilitaran el cambio hacia una administración de enfermería que debe estar acorde con los nuevos tiempos, donde se requiere mayor evidencia de impacto en la calidad del cuidado, en donde los lideres tienen un papel preponderante para poder guiar al personal operativo al logro de los objetivos institucionales, para supervisar y prevenir cualquier error.

En suma se puede decir que el perfil de liderazgo identificado va dirigido hacia una administración

Enf Neurol Vol. 15. No. 2 mayo - agosto 2016 . 89 
de manera eficiente y eficaz y que puede asumir las exigencias de cualquier situación en particular.

\section{CONCLUSIONES}

巨

I estudio cumplió con el objetivo analizar el perfil de liderazgo de los directivos de enfermería a fin de contar con un diagnóstico preciso que sirva como referente para la toma de decisiones

y como evidencia para estudios posteriores.

Sera necesario equililbrar el estilo de liderazgo y direccionarlo hacia las capacidades transformacionales, Se recomienda la capacitación continua de los directivos en temas relacionados con el liderazgo, a fin de mejorar el proceso de liderazgo y la formación del líder en enfermería y disminuir el perfil de Laissiez faire y conducirlo hacia el perfil transformacional.

\section{REFERENCIAS BIBLIOGRÁFICAS}

1. Aguilar Luzón, M. C.: Calvo-Salguero, A.; García-Hita, M. A. valores laborales y percepcion del estilo de liderazgo en personal de enfermería. Salud pública de México, 2007; 49(6): 401-407.

2. Vargas L. C. Organización de los servicios de Enfermería y sus niveles Jerárquicos. Subdirección General Médica del IMSS, disponible en: http:// bvs.insp.mx/artículos/2/10/05092001.pdt.

3. Rodríguez Marín, J. Estrés, Enfermedad y Hospitalización. Escuela Andaluza de Salud Pública. Monografías (17); 1997 p. 17- 25.

4. Telaranta, S. Opiniones sobre Gestión y Liderazgo en Enfermería Enfermería Global: 2007; 1(1): 1-74. Disponible en: http://www.scielo.php?pi$d=50717-9553301400020005$ scscript=sci_arttext.

5. Avolio B.J, Bass. B, M. Cuestionario de Liderazgo Multifactorial. Revista de Psicología del Trabajo y Organizacional; 1999 72, 447-462 Impreso en Gran Bretaña
6. Avolio, Bass. Re-examining the components of transformacional and transaccional Leadership using the Multifactor Leadership Questionnaire Journal of Occupational and Organizational Psychology: 1999 72, 441-462.

7. Reglamento de la Ley General de Salud en Materia de investigación para la Salud. (En línea). México: Cámara de Diputados H. congreso de la Unión; 1984 (consultado el 05-07-14) URL disponible en: http://www.sa/ud. gob.mx/unidades/cali/nom/compi/rlgsmis.html.

8. Milena R. Abaunza M. Liderazgo transaccional y transformacional. av enferm: 2010 jul-dic, 28(2) 62-72. Disponible en: hitp://wuw.indexf.com/ rae/282/062072.php

9. Berdecía CZ, González JR. Estilos de Liderazgo para el Éxito Organizacional: Estudio de Casos Múltiple en Empresas Puertorriqueñas Rev. De Estudios Avanzados de Liderazgo: 2013, 1(2) 2166-2320.

\section{ANEXOS}

Cuadro I: Perfil de liderazgo que ejerce la jefa de Enfermería. $n=6$

\begin{tabular}{|c|c|c|c|c|}
\hline \multirow{2}{*}{ Perfil/Tipo de liderazgo } & Transformación & Transaccional & \multicolumn{2}{|c|}{ Laissiez- faire } \\
\hline & № & № & _№ & $\%$ \\
\hline Bajo & 0 & 0 & 0 & 0 \\
\hline Medio & 67 & 50 & 2 & 33 \\
\hline Alto & 2 & 3 & 4 & 67 \\
\hline
\end{tabular}

Fuente: Multifactor Leadership Questionary (MLQ) "Visło por otro" 2015.

90 MNN/W4ht Enf Neurol Vol. 15. No. 2 mayo - agosto 2016 
Cuadro 2: Perfil de liderazgo que ejercen las supervisoras de Enfermería. $n=21$

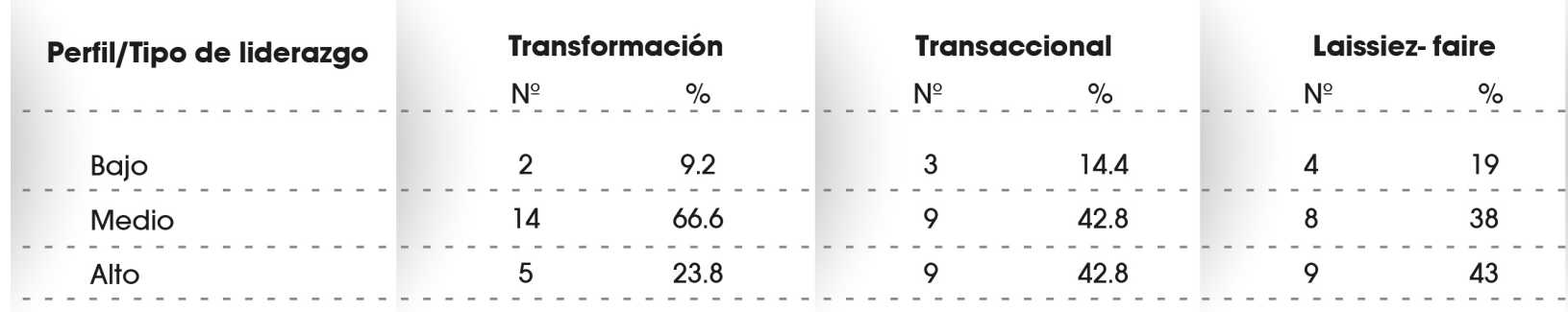

Fuente: Multifactor Leadership Questionary (MLQ) "Visto por otro" 2015.

Cuadro 3: Perfil de liderazgo que ejercen los jefes de servicio. $N=90$

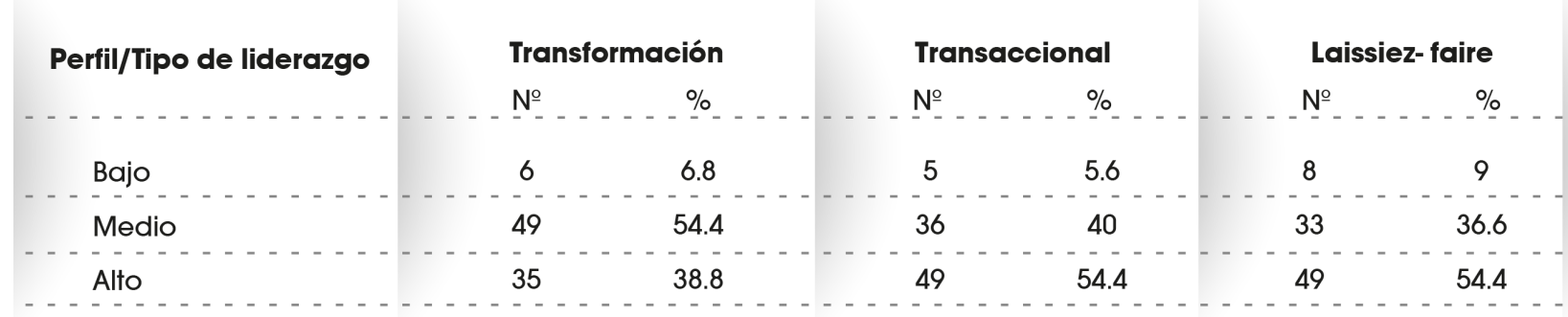

Fuente: Multifactor Leadership Questionary (MLQ) "Visto por otro" 2015.

Cuadro 4:Variables de resultado de los directivos de Enfermería. $n=117$

\begin{tabular}{|c|c|c|c|c|c|c|}
\hline \multirow{2}{*}{ Directivo de Enfermeria/Niveles } & \multicolumn{6}{|c|}{ Variables de resultado } \\
\hline & \multicolumn{2}{|c|}{ Esfuerzo Extra } & \multicolumn{2}{|c|}{ Efectividad } & \multicolumn{2}{|c|}{ Satisfacción } \\
\hline \multicolumn{7}{|l|}{ Jefa de Enfermeras } \\
\hline Bajo & 1 & 16.7 & 1 & 16.7 & 1 & 16.7 \\
\hline Medio & 1 & 16.7 & 3 & 50.0 & $i$ & 16.7 \\
\hline Alto & 4 & 67.0 & 2 & 33.0 & 4 & 67.0 \\
\hline Total & 6 & 100 & 6 & $100^{-}$ & 6 & 100 \\
\hline \multicolumn{7}{|l|}{ Supervisora de Enfermería } \\
\hline Bajo & 6 & 28.5 & 4 & 19.0 & 1 & 4.8 \\
\hline Medio & 9 & 43.0 & 8 & 38.0 & 10. & 47.6 \\
\hline Alto & 6 & 28.5 & 9 & 43.0 & 10 & 47.6 \\
\hline Total & 21 & 100 & 21 & 100 & 21 & 100 \\
\hline \multicolumn{7}{|l|}{ Jefe de Servicio } \\
\hline Bajo & 13 & 14.6 & 13 & 14.5 & 4 & 4.6 \\
\hline Medio & 33 & 36.6 & 41 & 45.5 & 33 & 36.6 \\
\hline Alto & 44 & 48.8 & 36 & 40.0 & 53 & 58.8 \\
\hline Total & 90 & 100 & 90 & 100 & 90 & 100 \\
\hline
\end{tabular}

Fuente: Multifactor Leadership Questionary (MLQ) "Visto por otro" 2015. 